\title{
ArcheoSciences
}

Revue d'archéométrie

\section{Minéralogie et signature isotopique du plomb des minerais auro-argentifères exploités durant l'époque romaine à Alburnus Maior (Rosia Montanà, Roumanie)}

Mineralogy and lead isotope signature of the gold-silver ores exploited during the Roman period at Alburnus Maior (Rosia Montanà, Romania)

Călin Gabriel Tămaş, Sandrine Baron et Béatrice Cauuet

\section{OpenEdition}

\section{Journals}

\section{Édition électronique}

URL : https://journals.openedition.org/archeosciences/2052

DOI : 10.4000/archeosciences.2052

ISBN : 978-2-7535-1598-7

ISSN : 2104-3728

Éditeur

Presses universitaires de Rennes

Édition imprimée

Date de publication : 31 décembre 2009

Pagination : 83-89

ISBN : 978-2-7535-1181-1

ISSN : 1960-1360

Référence électronique

Călin Gabriel Tămaş, Sandrine Baron et Béatrice Cauuet, « Minéralogie et signature isotopique du plomb des minerais auro-argentifères exploités durant l'époque romaine à Alburnus Maior (Rosia Montanà, Roumanie) », ArcheoSciences [En ligne], 33 | 2009, mis en ligne le 09 décembre 2012, consulté le 01 février 2022. URL : http://journals.openedition.org/archeosciences/2052 ; DOI : https:// doi.org/10.4000/archeosciences.2052 


\title{
Minéralogie et signature isotopique du plomb des minerais auro-argentifères exploités durant l'époque romaine à Alburnus Maior (Roşia Montană, Roumanie)
}

\author{
Mineralogy and lead isotope signature of the gold-silver ores exploited \\ during the Roman period at Alburnus Maior (Roşia Montană, Romania)
}

\author{
Călin Gabriel TĂMaş***,***, Sandrine Baron*** et Béatrice CaUUET ${ }^{* * *}$
}

\begin{abstract}
Résumé : Alburnus Maior (Roşia Montană, Roumanie), est un site minier important à l'époque romaine pour ses métaux précieux. Des études géologiques, gîtologiques et géochimiques ont été effectuées sur les corps de minerais exploités à l'époque romaine dans les chantiers miniers mis en évidence par l'archéologie minière. La démarche principale réside dans l'étude de minerais géo- et chrono- référencés par l'archéologie et la géologie pour identifier les sources des métaux précieux pendant l'antiquité romaine.

Les études géologiques menées dans le massif de Cârnic, ont permis d'identifier quatre phases de minéralisation. Trois d'entre elles ont été exploitées par les Anciens. Chacune se traduit par un corpus d'associations minéralogiques en métaux précieux dont deux d'entre elles possèdent des minéraux rares à tellures et à germanium. Par ailleurs, les teneurs élémentaires des autres éléments chimiques étant très hétérogènes, l’isotopie du plomb ( $\mathrm{Pb}$ ) est l'outil idéal pour optimiser la caractérisation. Chaque phase de minéralisation possède sa propre signature en $\mathrm{Pb}$. Malgré les différences isotopiques de $\mathrm{Pb}$ observées entre chacune d'elles, les signatures en $\mathrm{Pb}$ de Roşia Montană $(\mathrm{RM})$ constituent un champ isotopique très restreint. En comparaison avec d'autres districts miniers de Roumanie, le champ isotopique de RM est identifiable et affinera la filiation chimique à échelle régionale dans l'est de l'Europe.
\end{abstract}

\begin{abstract}
Alburnus Maior (Roşia Montană, Romania) was an important precious metals mining site during Roman times. Geological, ore deposit and geochemical studies have been carried out on the ore bodies exploited during the Roman period that have been discovered during archaeological excavations. The primary purpose of the present paper is to demonstrate the importance of the study of geologically and chronologically referenced ores by means of mining archaeology and geology for identifying the sources of precious metals from Roman Antiquity.

The geological studies carried out in the Cârnic Massif allowed the identification of four mineralisation phases. Three of these four phases have been exploited by the ancient miners. Each mineralised phase is characterised by a specific precious metals mineral association, and two of them contain rare Te and Ge bearing minerals. As their elemental composition is heterogeneous, the use of lead isotopes represents a good methodology for refining their characterisation. Each mineralised phase has its own lead signature. In spite of some observed differences in terms of lead isotopes for each phase, the overall signature of Roşia Montană (RM) displays a well defined isotopic field. When compared to other mining districts in Romania, the isotopic field of RM is distinguishable, and this allows a better discrimination that can contribute to the provenance study of minerals from Eastern Europe.
\end{abstract}

Mots-clés : Minerais Au-Ag, minéralogie, géochimie, isotopie, archéologie minière, mine romaine, Roşia Montană.

Keywords : Au-Ag ores, mineralogy, geochemistry, isotopes, mining archaeology, Roman mine, Roşia Montană.

* Université de Fribourg, Département de Géosciences, 6, Ch. du Musée, 1700 Fribourg, Suisse.

** Université Babeş-Bolyai - Faculté de Biologie et Géologie, 1, M. Kogălniceanu, 400084 Cluj-Napoca, Roumanie. (calin.tamas@ubbcluj.ro)

*** Laboratoire Travaux de Recherches Archéologiques sur les Cultures, les Espaces et les Sociétés, Université Toulouse 2 Le Mirail, CNRS UMR 5608 Maison de la Recherche, 5 allées Antonio-Machado, 31058 Toulouse cedex 09, France. 


\section{INTRODUCTION}

Roşia Montană (RM) est l'ancienne Alburnus Maior, une agglomération minière romaine mentionnée (entre autres) sur la plus ancienne des tablettes cirées (tabulae ceratae) retrouvées sur le site et datée du 6 février 131 (CIL TC XVII, Sîntimbrean, 1989), un quart de siècle après la conquête de la Dacie par les Romains. RM représente un gisement auro-argentifère de très grande taille exploité à ciel ouvert, mais également en souterrain dès l'époque romaine (Cauuet et al., 2003). À l'heure actuelle, RM reste le plus grand gisement de métaux précieux d'Europe (Manske et al., 2006) avec environ 410 tonnes d'or et 1840 tonnes d'argent pour des teneurs limites d'exploitation de $1,3 \mathrm{~g} / \mathrm{t}$ Au et 6 g/t Ag (www.gabrielresources.com; Jan 3, 2009). Un projet minier s'intéresse à ces ressources, il s'agit de la compagnie minière roumaine "Roşia Montana Gold Corporation ", une filiale de la compagnie canadienne "Gabriel Ressources". Dans le contexte d'une probable reprise minière du site de RM, des fouilles d'archéologie préventive se sont déroulées dans le cadre du Programme National de Recherche Alburnus Maior, sous la direction du Musée National d'Histoire de Roumanie. Jusqu'à ce jour, d'importantes fouilles archéologiques ont été achevées dans plusieurs secteurs miniers du site de RM (secteurs de Cetate et Cârnic) et d'autres fouilles sont actuellement en cours dans d'autres secteurs miniers de RM (secteurs Țarina, Orlea, Păru Carpeni, etc.) (Cauuet, sous presse). Létude présentée ici concerne le massif de Cârnic.

Une approche interdisciplinaire a été effectuée dans le cadre des fouilles archéologiques menées à RM. En effet, des études géologiques très détaillées de travaux miniers datant de l'époque romaine ont pu être effectuées grâce aux résultats fournis par l'archéologie minière. Nous avons donc eu la possibilité d'examiner et d'étudier les parois et les fronts de tailles creusés par les mineurs romains et ainsi les corps de minerais exploités réellement par les anciens mineurs. L'étude géologique de terrain a été poursuivie par des analyses minéralogiques et par un cortège d'analyses géochimiques. Le but a été d'établir une caractérisation minéralogique et géochimique de minerais auro-argentiferes archéologiques - c'est-à-dire effectivement exploités par les Anciens.

\section{LOCALISATION GÉOGRAPHIQUE ET CONTEXTE GÉOLOGIQUE}

Le gisement de RM fait partie du "Quadrilatère de l'or " (Ghițulescu et Socolescu, 1941), une région d'environ $900 \mathrm{~km}^{2}$ située dans la partie sud des Monts Apuseni
(Roumanie). Dans cette région, de nombreux gisements de porphyres cuprifères et des gisements épithermaux d'or et d'argent, liés au volcanisme néogène sont répertoriés, RM étant l'un des plus importants. Ce gisement (Fig. 1) est encaissé dans un flysch Crétacé supérieur, pénétré par trois épisodes volcaniques, dont le premier a été responsable de la mise en place des corps de métaux précieux de RM. Plus de détails sur la géologie de la région et sur les particularités du gisement de RM sont reportés ailleurs (Tămaş, 2007).

De plus, divers types de corps de minerai sont connus à l'échelle du gisement, il s'agit de filons, brèches, stockwerks, imprégnations, ainsi que placers et paléoplacers. Du point de vue minéralogique, les métaux précieux se présentent sous forme d'électrum, d'une grande variété de minéraux d'argent, de tellurures et de sulfures communs (Petrulian, 1934; Tămaş et al., 2004; 2006; Ciobanu et al., 2004).

\section{Matériels et MÉTHOdes}

Létude géologique a permis d'établir une cartographie géologique détaillée de tous les travaux miniers romains qui ont été fouillés dans le massif de Cârnic. Cette étude a permis d'effectuer la pétrographie des roches encaissantes, d'identifier les corps de minerais exploités par les Romains, ainsi que les altérations hydrothermales associées (notamment l'adularisation et la silicification), mais également les relations mutuelles entre divers corps de minerais. L'échantillonnage systématique (roches et minerais) et l'analyse des teneurs élémentaires des minerais effectuées au laboratoire Chemex (voir ci-dessous) ont complété l'étude géologique de terrain.

L'étude minéralogique des minerais issus de l'exploitation romaine a compris un examen microscopique (lames minces et lames polies), nécessaire pour l'identification des minéraux porteurs des métaux précieux et de minéraux associés. Des observations et analyses au microscope électronique à balayage (Jeol-JSM 6360 LV) ont permis de détailler la minéralogie des minéraux métalliques. Des analyses quantitatives effectuées sur les associations de minéraux métalliques ont été réalisées à l'aide d'une microsonde électronique de type CAMECA SX50 en utilisant une tension d'accélération de $25 \mathrm{kV}$, un courant d'analyse de $20 \mathrm{nA}$ et le diamètre du faisceau de $3 \times 3 \mu^{2}$. Des minéraux naturels et synthétiques, ainsi que des métaux purs et des alliages ont servi de matériaux de références.

Les analyses chimiques élémentaires ont été effectuées par Chemex, un laboratoire de service analytique de rang international, dont il n'est pas nécessaire de présenter ici toutes les caractéristiques, pour plus d'informations, se reporter au site web (www.alsglobal.com). 
Figure 1 : Géologie simplifiée du gisement de Roşia Montană. Dans l'encadré de la Roumanie, RM correspond au gisement de Roşia Montană et MM correspond au district de Maramureş, la partie ouest représente la région de Baia Mare et la partie est celle de Baia Borşa.

Figure 1: Simplified geology of Rossia Montană (RM). Inside the box representing Romania, $R M$ corresponds to the Roșia Montană ore deposit and MM corresponds to the Maramures metallogenetic district, which consists of the Baia Mare area in the west and the Baia Borşa area in the east.

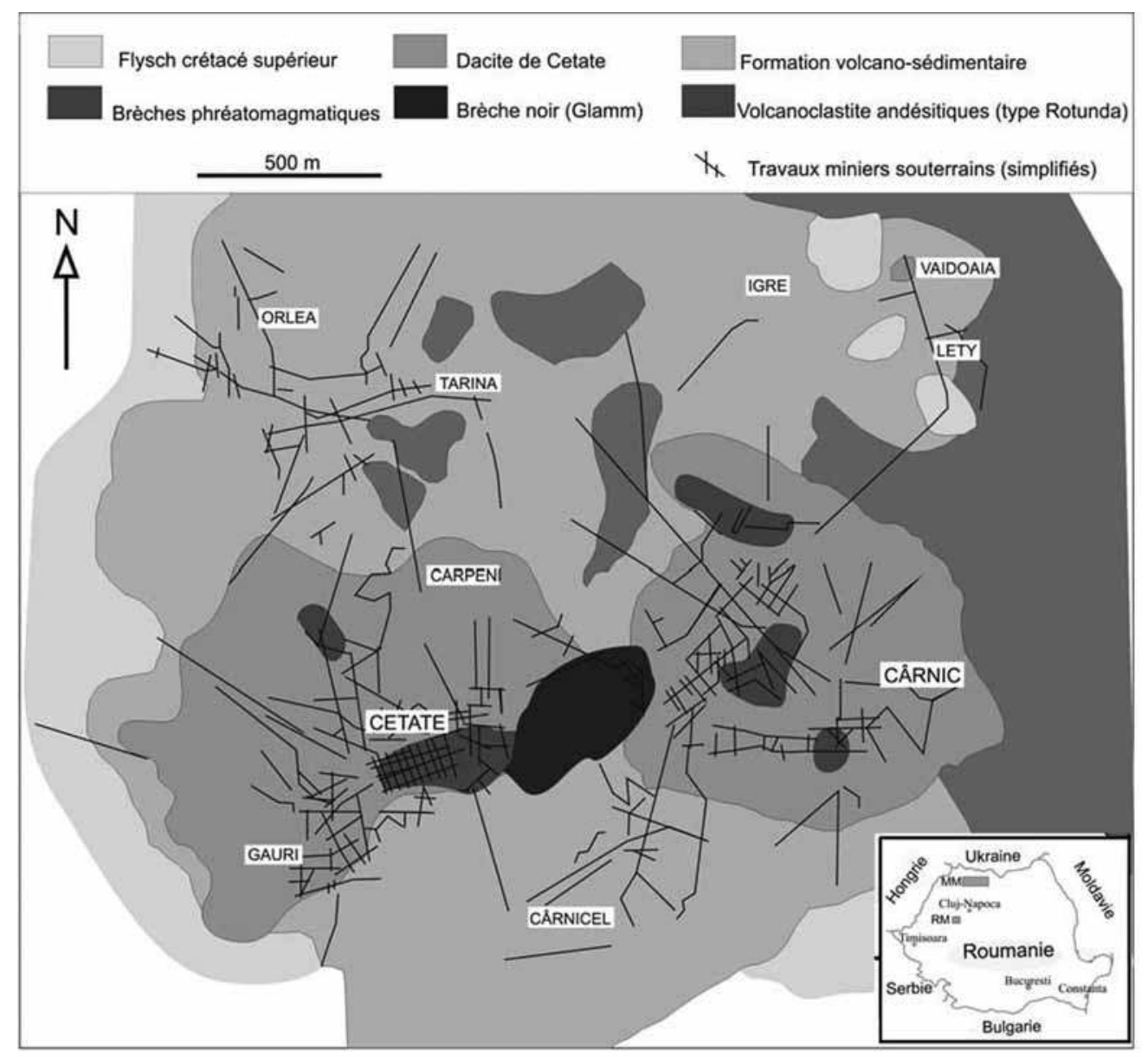

Les analyses isotopiques ont été conduites selon une méthode de préparation dont tous les détails ont été exposés antérieurement (Baron et al., 2006). L'appareil utilisé est un MC-ICP-MS (Multi-Collector inductively coupled plasma-mass spectrometer, Neptune, Thermo scientific). La méthode de mesure utilisée pour les rapports isotopiques du plomb $(\mathrm{Pb})$ est le dopage au thallium dont la présentation peut se retrouver dans la bibliographie (Baron et al., 2006). Le spectromètre de masse, équipé de neuf cages de Faraday, permet une mesure simultanée de tous les isotopes du plomb, du thallium et du mercure $\left({ }^{200} \mathrm{Hg}\right)$. Les mesures répétées du matériau de référence NIST NBS $981 \mathrm{~Pb}$ ont permis de calculer les valeurs de reproductibilité suivantes (2* écart type de la moyenne des valeurs du standard NIST NBS $981 \mathrm{~Pb}): 93 \mathrm{ppm}$ pour le rapport ${ }^{208} \mathrm{~Pb} /{ }^{206} \mathrm{~Pb}, 80$ ppm pour le rapport ${ }^{207} \mathrm{~Pb} /{ }^{206} \mathrm{~Pb}, 60 \mathrm{ppm}$ pour le rapport ${ }^{208} \mathrm{~Pb} /{ }^{204} \mathrm{~Pb}, 50 \mathrm{ppm}$ pour le rapport ${ }^{207} \mathrm{~Pb} /{ }^{204} \mathrm{~Pb}$ et $100 \mathrm{ppm}$ pour le rapport ${ }^{206} \mathrm{~Pb} /{ }^{204} \mathrm{~Pb}$.

\section{RÉsultats}

Les analyses géologiques et minéralogiques ont permis l'identification de différents types de corps de minerais exploités à l'époque romaine dans le massif de Cârnic (filons et corps de brèches). Ces corps de minerais correspondent à trois grandes phases de déposition de métaux précieux, chacune avec une minéralogie qui lui est propre, par conséquent des teneurs en métaux précieux spécifiques. Une quatrième phase de déposition a également été identifiée ailleurs dans les travaux miniers modernes du massif de Cârnic. Tandis que les trois premières phases ont été exploitées par les Romains, la quatrième phase métallogénique n’a pas été exploitée par les Anciens.

Les phases de déposition de minerais se caractérisent pour chacune par (Tableau 1) :

Phase \# 1 - Des structures de brèches phréatiques à ciment hydrothermal riche en quartz. Ce sont des corps de minerais très riches en or et argent (plus d'or que d'argent), avec une 


\begin{tabular}{|c|c|c|c|c|}
\hline \multirow{2}{*}{$\begin{array}{l}\text { Phase de } \\
\text { minéralisation }\end{array}$} & \multicolumn{2}{|c|}{ Composition minéralogique } & \multirow{2}{*}{ Éléments traces } & \multirow{2}{*}{ Observations } \\
\hline & Minéraux métalliques & Minéraux de gangue & & \\
\hline$\# 1$ & $\begin{array}{l}\text { électrum, polybasite, cuivre gris } \\
\text { argentifère, galène argentiferre, pyrite, } \\
\text { chalcopyrite et sphalérite; }\end{array}$ & $\begin{array}{l}\text { quartz, adulaire et chinga (ciment } \\
\text { riche en quartz et matière } \\
\text { organique); }\end{array}$ & - & $\begin{array}{l}\text { phase exploitée par les } \\
\text { mineurs romains }\end{array}$ \\
\hline$\# 2$ & $\begin{array}{l}\text { électrum, polybasite, pyrite, } \\
\text { chalcopyrite, sphalérite, cuivre gris, } \\
\text { marcasite et covellite; }\end{array}$ & quartz, adulaire; & - & $\begin{array}{l}\text { phase exploitée par les } \\
\text { mineurs romains }\end{array}$ \\
\hline \#3 & $\begin{array}{l}\text { achanthite, stéphanite, polybasite- } \\
\text { péarcéite, argent natif, cuivre gris } \\
\text { argentifere, galène argentifere, pyrite, } \\
\text { chalcopyrite, sphalérite, bornite, } \\
\text { électrum, argyrodite; traces de Te- } \\
\text { argyrodite et altaïte; }\end{array}$ & quartz, adulaire, chinga; & Ge, Te & $\begin{array}{l}\text { phase exploitée par les } \\
\text { mineurs romains }\end{array}$ \\
\hline$\# 4$ & $\begin{array}{l}\text { cuivre gris, galène, sphalérite, } \\
\text { chalcopyrite, pyrite, hessite (très } \\
\text { abondant), altaïte, sylvanite, Te- } \\
\text { argyrodite, électrum et marcasite. }\end{array}$ & $\begin{array}{l}\text { rhodonite, rhodochrosite, quartz, } \\
\text { adulaire. }\end{array}$ & $\mathrm{Te}, \mathrm{Ge}$ & $\begin{array}{l}\text { phase non exploitée par } \\
\text { les mineurs romains }\end{array}$ \\
\hline
\end{tabular}

Tableau 1 : Composition minéralogique des quatre phases de minéralisation mises en évidence dans le massif de Cârnic, Roşia Montana, Roumanie.

Table 1: Mineralogical composition of the four ores deposition phases from the Cârnic Massif, Roşia Montană, Romania.

composition minéralogique dominée par l'électrum et divers minéraux d'argent (sulfosels) avec des sulfures communs.

Phase \#2 - Des filons à gangue quartzeuse avec des teneurs très élevées en or et argent, mais avec des valeurs relativement similaires entre ces deux métaux précieux. Lélectrum et les minéraux d'argent (sulfosels) sont majoritaires, tandis que les sulfures communs sont très rares.

Phase \#3 - Des structures de brèches phréatiques rebréchifiées avec l'association de filons de quartz très riches en argent et des teneurs beaucoup plus faibles en or. La minéralogie de cette troisième phase de déposition est complexe avec une grande variété en sulfosels d'argent, argent natif, sulfure d'argent accompagnés d'électrum et de minéraux en base germanium, à savoir argyrodite $\left(\mathrm{Ag}_{8} \mathrm{GeS}_{6}\right)$ et argyrodite à tellure $\left(\mathrm{Ag}_{8} \mathrm{GeTe}_{2} \mathrm{~S}_{4}\right)$. Le tellure est systématiquement présent dans le sulfure d'argent (achantite) et la galène.

Phase \#4-Des filons à gangue rhodochrosite-rhodonite, avec plus de $1,15 \mathrm{~kg}$ d'argent et quelques grammes d'or à la tonne. La minéralogie de la quatrième phase est complètement différente des trois premières phases, avec des tellurures d'argent, d'or-argent et de plomb, de l'argyrodite à tellure, des sulfures communs et de l'électrum.

Les analyses chimiques élémentaires effectuées sur les minerais de chaque phase de minéralisation montrent des teneurs très hétérogènes, ce qui ne permet pas d'utiliser ces dernières en vue d'un éventuel traçage. Nous ne discuterons donc pas ces données. Néanmoins, les teneurs assez élevées en Te ( 250 ppm) et Ag (10 à > 1000 ppm) dans les minerais d'or de la phase 2 et 3 seront de bons traceurs élémentaires. En effet, comme il a été démontré par les études minéralogiques, les minéraux porteurs de ces éléments chimiques seraient une particularité des minerais de RM.

Les compositions isotopiques du plomb des quatre phases $(\varphi)$ de minéralisations sont reportées dans les graphiques de la Figure 2 (données en cours d'acquisition). Comme tous les rapports isotopiques montrent les mêmes tendances, seul le rapport isotopique ${ }^{206} \mathrm{~Pb} /{ }^{204} \mathrm{~Pb}$ sera pris en compte dans les discussions afin ne pas alourdir le texte. Chaque phase de minéralisation possède un champ de composition isotopique qui lui est propre (en dehors de l'incertitude totale externe des échantillons) et dont les différences sont significatives, à cette échelle (Fig. 2). Par contre, en moyenne, les quatre phases présentent un champ de compositions isotopiques très homogène. Par exemple, les valeurs moyennes, pour le rapport ${ }^{206} \mathrm{~Pb} /{ }^{204} \mathrm{~Pb}$, et pour chaque phase, sont les suivantes : $18.657 \pm 0.007$ ( $1 \sigma$ : écart type par rapport à la moyenne des valeurs isotopiques d'une phase donnée); $18.651 \pm 0.003(1 \sigma) ; 18.657 \pm 0.011(1 \sigma)$ et $18.635 \pm$ $0.001(1 \sigma)$ pour les phases $\varphi 1, \varphi 2$, $\varphi 3$ et $\varphi 4$ respectivement. Les Anciens ayant exploité uniquement trois des quatre phases, nous ne retiendrons que les compositions isotopiques de $\mathrm{Pb}$ de $\mathrm{RM}$ des phases $\varphi$ 1, $\varphi 2$ et $\varphi$ 3. Ainsi, la composition isotopique en $\mathrm{Pb}$ de Roşia Montană est une moyenne des trois phases, pour chaque rapport isotopique. La signature est, pour l'instant : $2.0778 \pm 0.0015(1 \sigma)$ pour le rapport ${ }^{208} \mathrm{~Pb} / 206 \mathrm{~Pb}, 0.8395 \pm 0.0004(1 \sigma)$ pour le rapport 

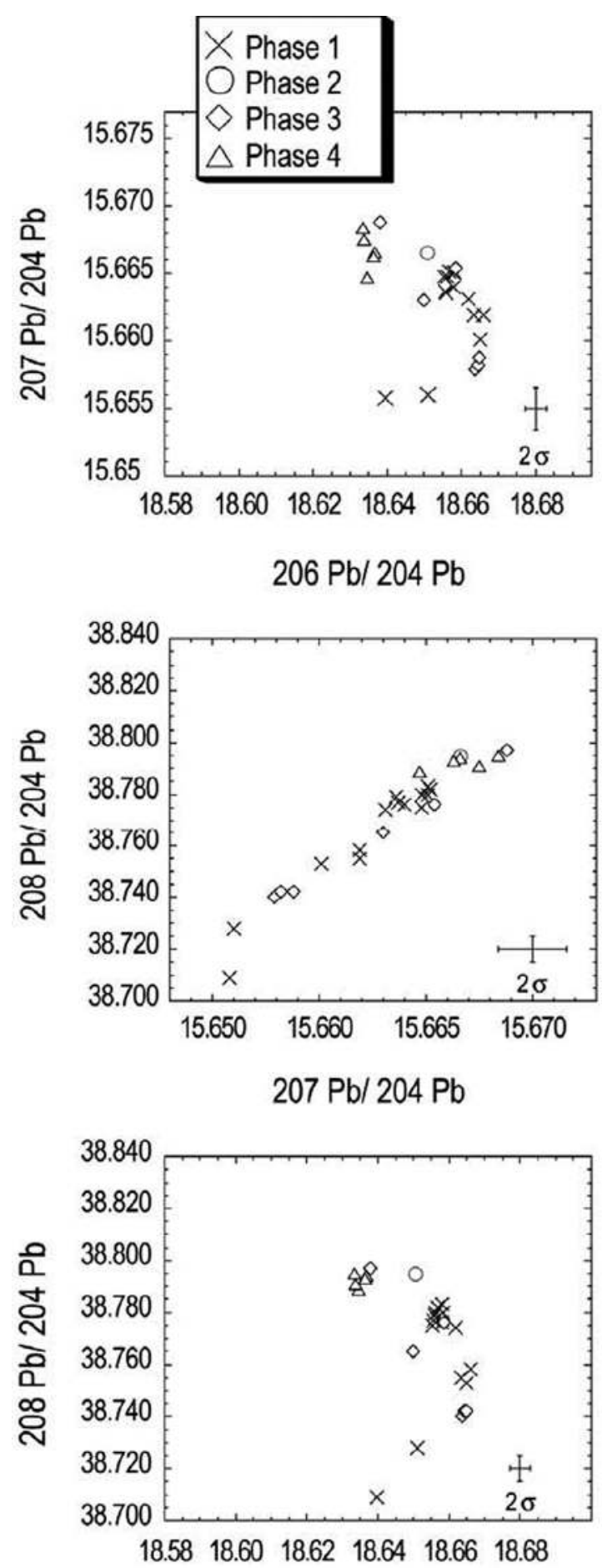

\section{$206 \mathrm{~Pb} / 204 \mathrm{~Pb}$}

Figure 2 : Diagrammes $\mathrm{Pb} / \mathrm{Pb}$ des quatre phases de minéralisations des minerais en Au-Ag du gisement de Roşia Montană, massif de Cârnic (Roumanie).

Figure 2: $\mathrm{Pb} / \mathrm{Pb}$ diagrams of the four $\mathrm{Au}-\mathrm{Ag}$ ore deposition phases from the Roşia Montană ore deposit, Cârnic Massif (Romania).
${ }^{207} \mathrm{~Pb} /{ }^{206} \mathrm{~Pb}, 38.765 \pm 0.023(1 \sigma)$ pour le rapport ${ }^{208} \mathrm{~Pb} /{ }^{204} \mathrm{~Pb}$, $15.663 \pm 0.004(1 \sigma)$ pour le rapport ${ }^{207} \mathrm{~Pb} / 204 \mathrm{~Pb}$ et $18.657 \pm$ $0.008(1 \sigma)$ pour le rapport ${ }^{206} \mathrm{~Pb} /{ }^{204} \mathrm{~Pb}$ (acquisitions finales des données en cours).

\section{Discussions}

Les analyses géologiques et minéralogiques de minerais exploités à l'époque romaine indiquent le caractère auroargentifère des deux premières phases de déposition et le caractère argentifère subordonné aurifère de la troisième phase. Dans ces trois phases de déposition, l'or apparait sous forme d'électrum et il est encaissé dans le ciment hydrothermal des brèches phréatiques, dans le quartz de filons et de brèches, ainsi que dans la pyrite. Mais, il est rarement inclus dans d'autres minéraux métalliques (sulfures et sulfosels). L'argent est concentré dans l'électrum et les sulfosels d'argent. En ce qui concerne la minéralogie de la troisième phase de déposition, nous devons souligner son caractère particulier en raison de l'occurrence de minéraux de germanium (argyrodite et argyrodite à tellure) et de la grande abondance de sulfosels d'argent avec une présence systématique du tellure. La quatrième phase de déposition, argentifere par excellence, mais non exploitée par les Romains dans le massif de Cârnic, s'individualise par la participation notable des tellurures $(\mathrm{Ag}, \mathrm{Ag}-\mathrm{Au}, \mathrm{Pb})$ et de l'argyrodite à tellure.

Les éléments chimiques retenant notre attention sont Ag, Te et Ge. De plus, une étude de fusion simple réalisée sur du minerai de RM (condition oxydante et réductrice avec une température $>1200{ }^{\circ} \mathrm{C}$ ) a montré que les teneurs en $\mathrm{Ag}$ et Te restent relativement identiques entre le minerai de départ et le minerai fondu (Hauptmann et al., 1995). Ag et Te ne semblent pas être affectés par le processus métallurgique. Par conséquent, $\mathrm{Ag}$ et Te seraient des éléments " conservatifs " dans ce cas précis, et ils seront de très bons indicateurs, en complément de l'isotopie du plomb, pour les futures études de traçabilité minerais - objets.

Les compositions isotopiques du plomb des minerais de $\boldsymbol{R} \boldsymbol{M}$. Sur ces diagrammes classiques $\mathrm{Pb} / \mathrm{Pb}$ (Fig. 2), les compositions isotopiques du plomb des minerais de Roşia Montană sont identifiables pour chacune des quatre phases déjà mises en évidence pas les études géologiques et minéralogiques. Chaque phase est l'illustration d'un évènement minéralisateur (mise en place de minerais) dont la source est un réservoir géologique plus ou moins profond et qui possède une composition isotopique qui lui est propre. Les compositions isotopiques sont assez homogènes et, outre le temps de mise en place assez court du district de RM, 
ces signatures en $\mathrm{Pb}$ indiquent surtout une source primaire commune (rapport U/Th identique et homogène) des différents fluides minéralisateurs, ce qui n'est pas forcément le cas dans les gisements métallifères. En effet, un seul et même gisement, ou une seule et même veine minéralisée, peut présenter des hétérogénéités isotopiques, ce qui peut perturber la filiation minerais-objets (Baron et al., 2006; 2009). Par conséquent, dans le cadre de problématiques archéologiques et historiques, il est indispensable de devoir caractériser un district minier d'un point de vue archéologique, géologique, mais aussi géochimique afin d'apporter des données pertinentes et complémentaires aux problématiques archéologiques et historiques, notamment sur la provenance des métaux.

\section{Le district de RM en contexte régional (à échelle de la Roumanie)}

Sur le graphique ${ }^{207} \mathrm{~Pb} /{ }^{204} \mathrm{~Pb}$ vs ${ }^{206} \mathrm{~Pb} /{ }^{204} \mathrm{~Pb}$ (Fig. 3), sont reportées les compositions isotopiques du plomb disponibles dans la littérature pour la Roumanie (Bird et al., 2008; Marcoux et al., 2002). Quatre groupes s'individualisent : le groupe des Monts Apuseni, les deux groupes de Maramures (celui de Baia Mare et de Baia Borşa) et Roşia Montană. Ce dernier groupe se distingue très bien au milieu du champ de compositions isotopiques de plomb des valeurs de minerais des Monts Apuseni au sens large.

Sur le diagramme de la Figure 3, nous pouvons observer que, globalement, les compositions isotopiques de $\mathrm{Pb}$ de la région de Maramures, au nord du pays, sont plus radiogéniques, notamment pour le rapport ${ }^{207} \mathrm{~Pb} /{ }^{204} \mathrm{~Pb}$, que celles des Monts Apuseni, au sud. La source minéralisatrice qui a conduit à ces gisements de Maramures est donc différente de celle du secteur des Monts Apuseni. Baia Borşa est le district minier qui possède la signature la moins radiogénique pour le rapport ${ }^{206} \mathrm{~Pb} /{ }^{204} \mathrm{~Pb}$ et de façon globale, c'est le secteur qui se distingue le plus des autres.

Par conséquent, au vu du graphique, chaque secteur minier est individualisable à échelle régionale. Par la suite, la traçabilité sera considérablement affinée dans le Nord/ Nord-Ouest de la Roumanie. Cela est prometteur dans la perspective des futures études de filiations des objets précieux antiques connus en Roumanie. En effet, nous pourrons mieux cerner géographiquement les aires d'extraction des minerais ayant servi à élaborer les objets en or découverts en Europe de l'Est au sens large.

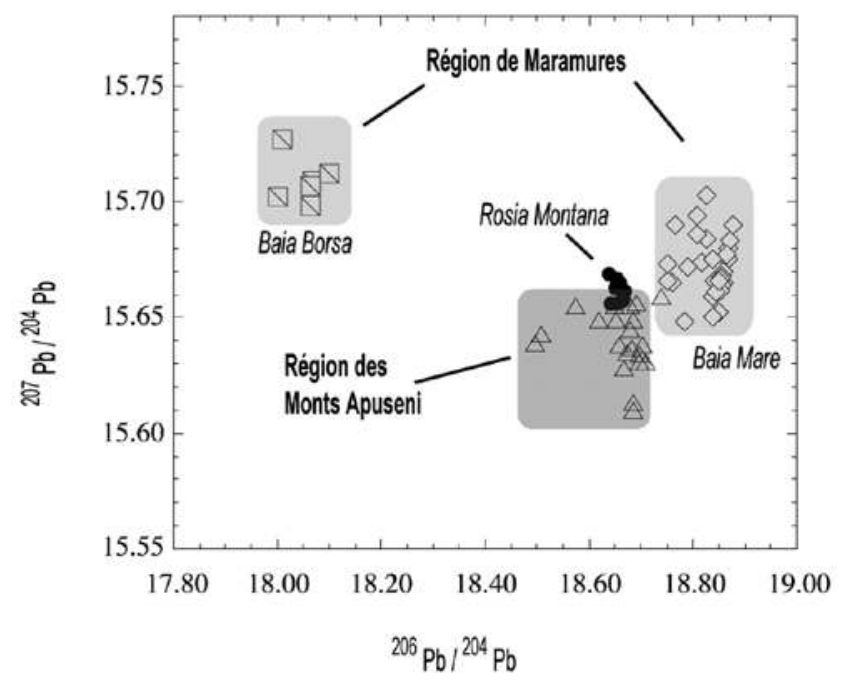

Figure 3 : Compositions isotopiques de plomb ${ }^{207} \mathrm{~Pb} /{ }^{204} \mathrm{~Pb}$ versus ${ }^{206} \mathrm{~Pb} /{ }^{204} \mathrm{~Pb}$ des districts miniers de Roşia Montană (inclus dans les Monts Apuseni), Baia Borşa et Baia Mare (région de Maramureş). Figure 3: ${ }^{207} \mathrm{~Pb}{ }^{204} \mathrm{~Pb}$ versus ${ }^{206} \mathrm{~Pb}{ }^{204} \mathrm{~Pb}$ ratios of the ore deposits from Roşia Montană (Apuseni Mountains), Baia Borşa and Baia Mare (Maramures district).

\section{Conclusions}

À Roşia Montană les études géologiques et minéralogiques menées récemment sur les travaux miniers mis au jour par l'archéologie minière ont permis de caractériser de façon précise les corps de minerais exploités pendant l'époque romaine. Sur les quatre phases de minéralisations mises en évidence par la géologie, trois phases ont été exploitées par les Romains. Elles se caractérisent par des teneurs élevées en $\mathrm{Ag}$ et Te, dont il sera possible de retrouver les traces (pour $\mathrm{Ag}$ et $\mathrm{Te}$ ) dans les objets précieux prochainement étudiés, étant donné que la réduction simple de minerai n'altère pas, a priori, les teneurs. L'isotopie du plomb, de haute résolution, effectuée sur ces matériaux archéologiques permet de proposer une signature de type "Roşia Montană ». La traçabilité des métaux précieux va être affinée à échelle régionale. La présente étude marque le point de départ de l'établissement d'une banque de données sur des matériaux archéologiques géo- et chrono- référencés. Cette étude est le fruit d'une démarche interdisciplinaire que nous souhaitons rendre systématique. 


\section{Remerciements}

Nous tenons à remercier la compagnie minière Roşia Montana Gold Corporation (RMGC) pour le soutien financier apporté non seulement aux études de terrain (exploration, fouilles archéologiques et géologie des travaux romains), mais aussi aux analyses minéralogiques et isotopiques. Le second auteur a bénéficié d'une bourse postdoctorale pour l'étude géochimique et isotopique. Les analyses $M E B$, microsondes électroniques et isotopiques ont été effectuées au Laboratoire des Mécanismes de Transferts en Géologie (LMTG-CNRS), Université Paul Sabatier, Toulouse, France.

\section{Bibliographie}

Bird, G., Brewer, P. A., Macklin, M. G., Balteanu, D., Serban, M., Driga, B. et Zaharia, S., 2008. River system recovery following the Novat-Rosu tailings dam failure, Maramures County, Romania, Applied Geochemistry 23: 3498-3518.

Baron, S., Mahé-Le-Carlier, C., Carignan, J. and Ploquin, A., 2009. Archaeological reconstruction of medieval lead production : Implications for ancient metal provenance studies and paleopollution tracing by $\mathrm{Pb}$ isotopes, Applied Geochemistry 24: 2093-2101.

Baron, S., Carignan, J., Laurent, S. et Ploquin, A., 2006. Medieval lead making on Mont-Lozère Massif (CévennesFrance) : tracing ore sources by using $\mathrm{Pb}$ isotopes, Applied Geochemistry 21: 241-252.

Cauuet, B., sous presse. L'espace minier romain. Le cas des mines d'or et d'argent d'Alburnus Maior en Dacie romaine (Roşia Montană, Roumanie), Actas del V Congreso de la Sociedad Espańola para la Protección del Patrimonio Geológico y Minero. Homenaje a Claude Domergue.

Cauuet, B., Ancel, B., Rico, Ch. et Tămaş, C., 2003. Ancient mining networks. The French archaeological missions 19992001. In Damian P. (dir.), Alburnus Maior Monographic series, I, Bucharest, p. 467-526.

Ciobanu, C.L., Cook, N.J, Tămaş, C., Leary, S., Manske, S., O’Connor, G. et Minuț, A., 2004. Telluride-gold-base metal associations at Roşia Montană : the role of hessite as gold carrier. In N.J. Cook, C.L. Ciobanu (ed.). Gold-silvertelluride deposits of the Golden Quadrilateral, South Apuseni Mts., Romania : guidebook of the International Field Workshop of IGCP project 486, Alba Iulia, Romania, 31 $1^{\text {st }}$ August $-7^{\text {th }}$ September 2004, (IAGOD Guidebook Series; 12), 187-202.

Ghițulescu, T.P. et Socolescu, M., 1941. Etude géologique et minière des Monts Métallifères (Quadrilatère aurifère et régions environnantes), Annuaire Institute Géologique Roumain XXI: $181-465$.

Hauptmann, A, Rehren, Th. et Pernicka, E., 1995. The composition of gold from the ancient mining district of Verespatak/ Roşia Montana, Romania. In G. Morteani, J.P. Northover (ed.), Prehistoric Gold in Europe, p. 369-381.

Manske, S.L., Hedenquist, J.W., O’Connor, G., TĂmaş, C., Cauuet, B., Leary, S. et Minuț, A., 2006. Roşia Montană, Romania : Europe's largest gold deposit, Society of Economic Geologists Newsletter 64: 1, 9-15.

Marcoux, E., Grancea, L., Lupulescu, M. et Milési, J. P., 2002. Lead isotope signatures of epithermal and prophyrytype ore deposits from the Romanian Carpathian Mountains, Mineralium Deposita, 37, p. 173-184.

Petrulian, N., 1934. Étude chalcographique du gisement aurifère de Roşia Montană (Transylvanie, Roumanie), Annuaire Institute Géologique Roumain XVI: 499-539.

Sîntimbrean, A., 1989. Muzeul mineritului din Roşia Montană, Editura Sport Turism, Bucureşti, 219 p. (en roumain).

Tămaș, C.G., Bailly, L., Ghergari, L., O’Connor, G. et Minuț, A., 2004. First reported tellurides occurrence in Roșia Montană, Apuseni Mountains, Romania, 32 $2^{\text {nd }}$ International Geological Congress Abstract Volume, Florence, Italy, August 20-28: 273.

Tămaş, C.G, Bailly, L., Ghergari, L., O'Connor, G. et Minuț, A., 2006. New telluride and argyrodite occurrences in Roşia Montană, Apuseni Mountains, Romania and their metallogenic significance, Canadian Mineralogist, 44: 689-705.

Tămaş, C.G., 2007. Structuri de brecii endogene (breccia dyke breccia pipe) şi petrometalogenia zăcământului Roşia Montană (Munții Metaliferi, România), Ed. Casa Cărții de Știință, ClujNapoca, 230 p. (en roumain). 
